\title{
Spontaneous Intramural Intestinal Hemorrhage versus Acute Mesenteric Ischemia by CT Evaluation
}

\author{
Chia-Ying Tseng ${ }^{1,2}$, Chia-Ming Chang ${ }^{2,3}$, Shu-Chuan Yang ${ }^{3}$, Julia Chia-Yu Chang ${ }^{2,3}$, \\ Jen-Dar Chen ${ }^{3,4}$, Hsien-Hao Huang ${ }^{2,3,5}$, Chorng-Kuang How ${ }^{2,3,5}$, \\ David Hung-Tsang Yen ${ }^{2,3,5}$ and Mu-Shun Huang ${ }^{2,3}$
}

\begin{abstract}
Objective The purpose of our study was to differentiate the imaging findings of patients with spontaneous intramural intestinal hemorrhage (SIIH) from those with acute mesenteric ischemia (AMI) after abdominal computed tomography (CT) survey in the emergency department.

Methods We retrospectively included 83 patients diagnosed with SIIH or AMI after abdominal CT.

Results The mean ages of $30 \mathrm{SIIH}$ patients and 53 AMI patients were $74.4 \pm 14.6$ years and $75.8 \pm 11.2$ years, respectively. Patients with SIIH had significantly thicker maximal intestinal wall thickening $(14.8 \pm 3.9$ vs. $10.9 \pm 4.1, \mathrm{p}<0.001)$, a lower rate of ileum involvement $(26.7 \%$ vs. $77.4 \%, \mathrm{p}<0.001)$ and a higher rate of ascites $(96.7 \%$ vs. $64.2 \%, \mathrm{p}<0.001)$ compared with patients with AMI. Neither pneumatosis intestinalis $(\mathrm{p}<$ $0.001)$ nor portomesenteric gas $(\mathrm{p}<0.01)$ were detected in SIIH patients but were observed in AMI patients. A receiver-operating characteristic (ROC) curve analysis showed that the optimal cut-off value for maximal intestinal wall thickening between groups was $10.4 \mathrm{~mm}$ and the area under the ROC curve between groups was $0.752(\mathrm{p}<0.0001)$. A multiple logistic regression analysis showed that the independent predictors of SIIH were non-involvement of the ileum (odds ratio, OR, 6.998; $\mathrm{p}=0.001$ ), maximal intestinal wall thickening $\geq 10.4 \mathrm{~mm}$ (OR, 5.748; $\mathrm{p}=0.040)$ and ascites (OR, 13.348; $\mathrm{p}=0.023)$. The area under the ROC curve for the model was $0.854(\mathrm{p}<0.001)$.

Conclusion The independent predictors of SIIH from AMI after abdominal CT in acute abdominal patients include non-involvement of the ileum, intestinal wall thickening $\geq 10.4 \mathrm{~mm}$, and ascites.
\end{abstract}

Key words: spontaneous intramural intestinal hemorrhage, acute mesenteric ischemia, maximal intestinal wall thickening, non-involved ileum

(Intern Med 55: 2337-2341, 2016)

(DOI: 10.2169/internalmedicine.55.6772)

\section{Introduction}

Spontaneous intramural intestinal hemorrhage $(\mathrm{SIIH})$ is a rare, acute abdomen condition, occurring with increasing frequency as a complication of warfarin anticoagulation overuse in emergency departments (EDs). Patients typically complain of abdomen pain $(100 \%)(1,2)$, and the rate of peritonitis is approximately $7.7-61.5 \%$ (1-3). Emergency physicians (EPs) must exclude the possibility of surgical intervention. Abdominal computed tomography (CT) can help EPs to differentiate patients with acute abdominal pain due to its characteristics of rapid, first-line, imaging modality with high accuracy. However, abdominal CT occasionally is not useful to help EPs differentiate SIIH from acute mesenteric ischemia (AMI) due to similar findings, such as homogenous bowel wall thickening or the presence of a target sign on intravenous contrast-enhanced studies (4-6). Ap-

\footnotetext{
${ }^{1}$ Department of Emergency Medicine, Taichung Veterans General Hospital, Taiwan, ${ }^{2}$ Emergency Medicine, College of Medicine, National YangMing University, Taiwan, ${ }^{3}$ Department of Emergency Medicine, Taipei Veterans General Hospital, Taiwan, ${ }^{4}$ Department of Radiology, Taipei City Hospital, Taiwan and ${ }^{5}$ Institute of Emergency and Critical Care Medicine, College of Medicine, National Yang-Ming University, Taiwan Received for publication November 4, 2015; Accepted for publication December 14, 2015 Correspondence to Dr. Hsien-Hao Huang, hhhuang@vghtpe.gov.tw
} 
Table 1. Demographic Information and Comorbid Medical in Patients with SIIH or AMI at ED.

\begin{tabular}{lccc}
\hline & $\begin{array}{c}\text { SIIH } \\
(\mathrm{n}=30)(\%)\end{array}$ & $\begin{array}{c}\text { AMI } \\
(\mathrm{n}=53)(\%)\end{array}$ & $\mathrm{p}$ \\
\hline Sex (male : female) & $23: 7$ & $33: 20$ & $\mathrm{NS}$ \\
Age (years) & $74.4 \pm 14.6$ & $75.8 \pm 11.2$ & $\mathrm{NS}$ \\
Duration before ED visits (days) & $2.5 \pm 1.4$ & $2.5 \pm 1.9$ & $\mathrm{NS}$ \\
Anticoagulant use & $28(93.3)$ & $5(9.4)$ & $<0.001$ \\
Surgery & $0(0)$ & $33(62.3)$ & $<0.001$ \\
Mortality & $2(6.7)$ & $33(62.3)$ & $<0.001$ \\
\hline
\end{tabular}

proximately $17.9 \%$ of SIIH patients are transferred to medical centers for surgical intervention under the impression of AMI (2). Furthermore, approximately $32.1 \%$ of SIIH patients' formal abdominal CT reports by radiologists suggest the need for EPs to differentiate SIIH from AMI in clinical practice (2). It is critical for EPs to differentiate $\mathrm{SIIH}$ from AMI because the treatments are quite different, i.e., conservative medical treatment for $\operatorname{SIIH}(1,3,7,8)$ and emergent surgical intervention for AMI (9-11). The purpose of our study was to discern the differences in abdominal CT findings and help EPs to differentiate SIIH from AMI.

\section{Materials and Methods}

We retrospective reviewed the emergency medical charts of patients admitted to Taipei Veterans General Hospital from 2003 to 2013, which has an ED that sees approximately eighty thousand visits annually. Fifty-three cases that underwent abdominal CT in the ED were identified from a computer search of our hospital discharge codes (International Classification of Disease, ICD) of 557.0 and 557.9. The definition of AMI was bowel necrosis due to 4 types of pathophysiologic processes (12), including: 1) mesenteric arterial emboli (incidence 40-50\%) from a cardiac source; 2) mesenteric arterial thrombosis $(25 \%)$ due to severe atherosclerotic disease; 3) non-occlusive mesenteric ischemia (20\%) due to a low cardiac output; and 4) mesenteric venous thrombosis $(10 \%)$ due to an intraabdominal pathological condition or idiopathic. Initial resuscitation included intravenous fluid resuscitation, therapeutic intravenous heparin sodium and mesenteric arterial infusion of papaverine. Emergency laparotomy is typically needed to explore the possibility of bowel infarction and excision, except in nonocclusive mesenteric ischemia. The treatment for nonocclusive mesenteric ischemia is primarily medical conservative therapy. The diagnosis of AMI was made by surgical intervention (31/53) and an angiogram (6/53) and abdominal CT (53/53) with diagnostic criteria including bowel wall thickening, appearance of a target sign, lack of an enhanced bowel wall, pneumatosis intestinalis, arterial emboli and venous thrombosis. In AMI, patients with mesenteric ischemia secondary to mechanical obstruction, adhesions, volvulus, or clinically diagnosed AMI without abdominal CT were excluded, as were patients younger than 14 years of age. Thirty cases of SIIH that underwent abdominal CT in the
ED were identified of ICD 569.8 and 578. For the diagnosis of SIIH, the discharge diagnosis and non-traumatic spontaneous intramural hematoma after abdominal CT survey of homogenous bowel wall thickening, enhanced target sign and highly attenuated ascites were reviewed (30/30). The final diagnosis of SIIH was determined by abdominal CT (30/ $30)$ and a history of warfarin use (28/30) with prolonged activated partial thromboplastin time or hemophilia A (2/30). Two trained research assistants blinded to the study purpose entered the abstracted data into a new data bank and standardized abstractor forms used for study analyses. The findings of a CT scan, such as involved segment of the intestine, maximal intestinal wall thickening, bowel segments and the estimated length involved, ascites, pneumatosis intestinalis, portomesenteric gas, and mesenteric arterial or venous thrombosis were evaluated and analyzed by two abdominal radiologists who were blinded to the clinical diagnosis of AMI or SIIH. Categorical variables were compared between groups using the chi-square or Fisher's exact test for qualitative data. The Mann-Whitney non-parametric U-test or a $t$ test was used to analyze quantitative data. Multiple logistic regression analyses were applied to determine the accuracy of combinations of clinical significant variables in predicting patient outcomes. The Hosmer-Lemeshow test for lack of fit and likelihood ratio test used for model selection were performed. When applicable, tests were two-tailed. $\mathrm{p}$ values $<0.05$ were considered to be statistically significant.

\section{Results}

Among the 83 patients studied, 30 (23 men and 7 women) had SIIH and 53 ( 33 men and 20 women) had AMI. The mean ages were $74.4 \pm 14.6$ years (range, 18-93 years) and 75.8 \pm 11.2 years (range, 38-95 years), respectively (Table 1). As compared with AMI patients, all included SIIH patients received conservative medical treatment without surgery $(\mathrm{p}<0.001)$. Only $2(6.7 \%)$ SIIH patients died due to pneumonia and septic shock $(\mathrm{p}<0.001)$. In SIIH patients, anticoagulant use $(28 / 30,93.3 \%)$ was the major cause, while the other two patients had hemophilia A and suspected pancreatitis-induced coagulopathy. In AMI patients, there were $5(9.4 \%)$ patients under anticoagulant treatment.

All included patients were diagnosed with SIIH or AMI after an abdominal CT examination in our ED. In the final formal reports, only $66.7 \%$ (20/30) suggested the diagnosis of SIIH alone, $23.3 \%$ (7/30) suggested that EPs must differentiate SIIH from AMI, and 10.0\% (3/30) suggested the diagnosis of AMI alone (Table 2). Thus, the purpose of our study was to discern the differences in abdominal CT findings to differentiate SIIH from AMI.

With regard to the abdominal CT findings (Table 3), there was no difference between SIIH and AMI ( $p>0.05)$ in the degree of jejunum involvement or the estimated length. Maximal intestinal wall thickening was significantly thicker in SIIH patients as compared with AMI patients (14.8 \pm 3.9 vs. 10.9 $\pm 4.1, \mathrm{p}<0.001)$. The percentage of involved intestine 
Table 2. The Formal Abdominal CT Reports in SIIH and AMI Suggested the Differential Diagnosis in Our ED.

\begin{tabular}{lcc}
\hline & $\begin{array}{c}\text { SIIH } \\
(\mathrm{n}=30)(\%)\end{array}$ & $\begin{array}{c}\text { AMI } \\
(\mathrm{n}=53)(\%)\end{array}$ \\
\hline SIIH only & $20(66.7)$ & $0(0)$ \\
Differentiate SIIH from AMI & $7(23.3)$ & $0(0)$ \\
AMI only & $3(10)$ & $53(100)$ \\
Enteritis or colitis & $5(16.7)$ & $8(15.1)$ \\
Partial intestinal obstruction & $1(3.3)$ & $4(7.5)$ \\
Acute pancreatitis & $1(3.3)$ & $0(0)$ \\
\hline * 8 SIIH patients and 16 AMI patients have more than one
\end{tabular}

differential diagnosis in formal abdominal CT reports

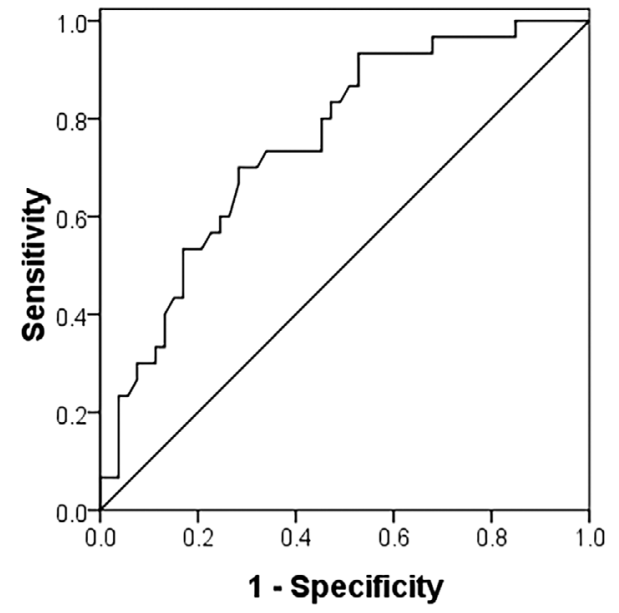

Figure 1. A ROC curve of the cut-off level of maximal intestinal wall thickening to differentiate SIIH from AMI.

was approxiamately $10.0 \%(\leq 15 \mathrm{~cm})$ and $46.7 \%(16-30 \mathrm{~cm})$ in SIIH patients (mean $33.2 \mathrm{~cm}$ ), and $50.9 \%(>30 \mathrm{~cm})$ in AMI patients (mean $36.7 \mathrm{~cm})(\mathrm{p}>0.05)$. SIIH patients also had a significant lower rate of ileum involvement $(26.7 \%$ vs. $77.4 \%, \mathrm{p}<0.001)$ and a significant higher rate of ascites $(96.7 \%$ vs. $64.2 \%, \mathrm{p}<0.001)$. Neither pneumatosis intestinalis nor portomesenteric gas was found in all SIIH patients, and these were only identified in AMI patients, at rates of $39.6 \%$ (21/53) and $20.8 \%$ (11/53), respectively. Intravenous medium aids in the detection of mesenteric arterial or venous thrombosis in abdominal CT. In our study, $100 \%$ (30/ 30) of SIIH patients and $75.5 \%$ (40/53) AMI patients underwent abdominal CT with intravenous contrast medium. The rates of mesenteric arterial or venous thrombosis in $\mathrm{SIIH}$ and AMI patients were $0 \%(0 / 30)$ and $52.5 \%$ (21/40), respectively.

A receiver-operating characteristic (ROC) curve analysis was performed to determine the potential role of maximal intestinal wall thickening in the discrimination between the SIIH and AMI groups. The area under the ROC curve between groups was 0.752 (95\% confidence interval, 0.6460.857; p<0.0001) (Fig. 1). The optimal cut-off value for maximal intestinal wall thickening between the groups was $10.4 \mathrm{~mm}$. When maximal intestinal wall thickening was $\geq 10.4 \mathrm{~mm}$, the sensitivity, specificity, positive predictive
Table 3. Summary of Abdominal CT Findings in SIIH and AMI at ED.

\begin{tabular}{|c|c|c|c|}
\hline & $\begin{array}{c}\text { SIIH } \\
(n=30)(\%)\end{array}$ & $\begin{array}{c}\text { AMI } \\
(\mathrm{n}=53)(\%)\end{array}$ & $\mathrm{p}$ \\
\hline Duodenum involved $^{\mathrm{a}}$ & $1(3.3)$ & $0(0)$ & NS \\
\hline Jejunum involved & $23(76.7)$ & $37(69.8)$ & NS \\
\hline Ileum involved & $8(26.7)$ & $41(77.4)$ & $<0.001$ \\
\hline Maximal intestinal wall thickening $(\mathrm{mm})^{\mathrm{b}}$ & $14.8 \pm 3.9$ & $10.9 \pm 4.1$ & $<0.001$ \\
\hline Estimated length $(\mathrm{cm})$ & $33.2 \pm 19.4$ & $36.7 \pm 27.4$ & NS \\
\hline Ascites & $29(96.7)$ & $34(64.2)$ & 0.001 \\
\hline Pneumatosis intestinalis & $0(0)$ & $21(39.6)$ & $<0.001$ \\
\hline Portomesenteric gas & $0(0)$ & $11(20.8)$ & $<0.01$ \\
\hline Mesenteric arterial or venous thrombosis ${ }^{c}$ & $0 / 23(0)$ & $21 / 40(52.5)$ & $<0.001$ \\
\hline
\end{tabular}

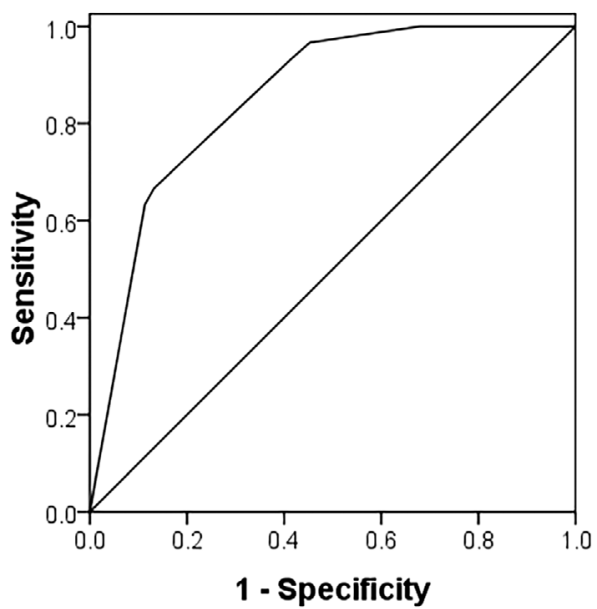

Figure 2. A ROC curve displaying the relationship between the sensitivity and specificity for the regression-model analysis of independent variables predicting SIIH from AMI after abdominal CT.

value (PPV) and negative predictive value (NPV) were $93.3 \%, 45.3 \%, 49.1 \%$ and $92.3 \%$, respectively.

We conducted a multiple logistic regression analysis by entering backward and stepwise data for increased ileum involvement, ascites and whether intestinal wall thickening exceeded and included $10.4 \mathrm{~mm}$. The independent predictors of SIIH were non-involvement of the ileum, (odds ratio, OR, 6.998; $95 \%$ confidence interval, $\mathrm{CI}, 2.180$ to 22.457 ; $\mathrm{p}=$ 0.001 ), maximal intestinal wall thickening $\geq 10.4 \mathrm{~mm}$ (OR, 5.748; 95\% CI, 1.084 to $30.477 ; \mathrm{p}=0.040)$ and ascites (OR, 13.348; 95\% CI, 1.433 to $124.357 ; \mathrm{p}=0.023)$. The area under ROC curve (Fig. 2) for the model was 0.854 (95\% CI, 0.773 to $0.934 ; \mathrm{p}<0.0001)$, suggesting good model discrimination. The sensitivity, specificity, PPV and NPV were $63.3 \%, 86.7 \%, 76.0 \%$ and $81.0 \%$, respectively.

\section{Discussion}

SIIH is rare in the list of differential diagnoses, however, with the increasing overuse of warfarin for anticoagulation, SIIH is being reported more frequently. Unfortunately, abdominal CT occasionally does not help EPs differentiate 
SIIH from AMI due to similar CT findings (4-6). It is critical for EPs to make the right decision because the optimal treatments are quite different, conservative medical treatment for $\operatorname{SIIH}(1,7,8)$ and emergent surgical intervention for AMI (9-11). In our study, 20\% (6/30) of the SIIH patients were transferred to our hospital under the impression of AMI and for emergent surgical intervention. Approximately $33.3 \%$ (10/30) of our hospital formal abdominal CT reports for SIIH patients suggested AMI or the possibility of AMI (Table 2). In order to help EPs to differentiate SIIH from AMI by discerning the differences in abdominal CT findings, our retrospective study showed that the independent predictors of SIIH were non-involved ileum, intestinal wall thickening $\geq 10.4 \mathrm{~mm}$ and ascites.

The jejunum was the most commonly involved site of hematoma in SIIH patients (13). In previous studies of SIIH, the rates of intestinal segments involved were as follows: $50-69 \%$ in the jejunum, 38-61.1\% in the ileum, and 5.6$23 \%$ in the duodenum $(3,14,15)$, while in the present study they were $76.7 \%, 26.7 \%$ and $3.3 \%$, respectively. In AMI, the ileum was the most common involved (84.2\%), followed by the jejunum $(47.4 \%)$, duodenum $(10.5 \%)$, and colon (5.3\%) (14). According to the above findings, the jejunum was the most common involved part of the intestine in SIIH $(3,15)$, whereas it was the ileum in AMI (14). The invovlement of the jejunum may help EPs to differentiate SIIH from AMI. In our study, the involvement of the jejunum did not show significant difference between SIIH and AMI ( $p>0.05)$, whereas the involvement of the ileum did. The rate of ileum involvement was significantly lower in SIIH patients $(\mathrm{p}<0.001$, Table 3$)$. A multiple logistic regression analysis found that non-involvement of the ileum was an independent predictor of SIIH from AMI after abdominal CT. Non-involvement of the ileum may help clinical physicians to differentiate SIIH from AMI patients.

Wall thickening of the involved intestine was easily detected and estimated from CT. The mean maximal wall thickening of the involved intestine in SIIH was approximately $11.7-14.1 \mathrm{~mm}(7,14)$ and $14.8 \mathrm{~mm}$ in our study. Regarding AMI, the mean maximal wall thickening was about $4 \mathrm{~mm}$ (14) and $10.9 \mathrm{~mm}$ in our study. The maximal intestinal wall thickening in SIIH was thicker than that in AMI (p $<0.001$ ) (14), which is consistent with our study $(\mathrm{p}<0.001)$. Whether maximal wall thickening of the involved intestine is greater or less than $10 \mathrm{~mm}$ has been previously proposed as a means to differentiate SIIH from AMI (14). Our study analyzed the abdominal CT findings of $30 \mathrm{SIIH}$ patients, the largest series to date, and used statistical analyses to determine the cut-off value of maximal wall thickening. It has been shown that maximal wall thickening can indeed discriminate SIIH from AMI $(\mathrm{p}<0.0001)$ and a thickness greater than $10.4 \mathrm{~mm}$ implies SIIH. A multiple logistic regression analysis found that maximal wall thickening $\geq 10.4$ $\mathrm{mm}$ was an independent predictor of SIIH from AMI.

The estimated length of the involved intestine was previously discussed between SIIH and AMI. The percentage of involved intestine was approximately $78 \%(\leq 15 \mathrm{~cm})$ and $22 \%(16-30 \mathrm{~cm})$ in SIIH patients, and $84 \%(>30 \mathrm{~cm})$ in AMI patients (14). The estimated length of the involved intestine was significant longer in AMI than SIIH (14). However, in our study, there was no significant difference between SIIH and AMI patients. Perhaps due to advances in CT imaging and the early detection of AMI, the range and mean estimated length of involved intestines in AMI was 5$128 \mathrm{~cm}$ and $36.7 \mathrm{~cm}$, respectively, which was not significant longer than that of SIIH.

Anticoagulant use history was also significantly different between SIIH and AMI $(\mathrm{p}<0.001)$. We analyzed a subgroup of patients under anticoagulant treatment (28 SIIH patients vs. 5 AMI patients), however, there were no significant difference in sex, age, duration before ED visits or abdominal CT findings. Only ascites exhibited a significant difference between SIIH and AMI (27/28 (96.4\%) vs. 2/5 (40.0\%), p= 0.007).

Pneumatosis intestinalis (PI) is defined as the presence of gas in the bowel wall and CT has a high imaging sensitivity to identify intramural gas (16). PI can be detected in acute surgical $(17,18)$ and non-surgical abdomen disease (17-20). For AMI, the rate of PI in CT reports in previous studies was approximately $26.7-60 \%(17,21)$ and $39.6 \%$ in our study. For SIIH, PI was not found in the CT reports in previous studies $(1,3,5,7,14,15)$ or in our study. Although SIIH had a significantly lower rate of PI as compared with AMI $(p<0.001)$, we could not analyze the odds ratio of PI since it was $0 \%$ in SIIH patients. Therefore, PI may help EPs to differentiate SIIH from AMI, however, more studies are needed to confirm this.

Hepatic portal venous gas (HPVG) is also easily detected in CT and associated with either benign medical disease (e. g., ulcerative colitis, intraabdominal abscess and gastric ulcer) or lethal surgical diseases (e.g., necrotic bowel and small bowel obstruction) (22). Approximately 16.1-35.7\% of AMI patients had HPVG in previous studies $(16,21)$ and $20.8 \%$ in our study, however, HPVG showed no significant difference between the survivors and non-survivors in AMI patients (21). Regarding SIIH, HPVG was not found in previous studies $(1,3,5,7,14,15)$. A greater accumulation of data is needed to validate the effect of HPVG in discriminating between SIIH and AMI.

\section{Study limitations}

The strengths of this study include the large number of SIIH patients and statistical analyses for prognostic indicators to differentiate SIIH from AMI. There are some limitations associated with this study. First, this is a retrospective study and was limited to only a tertiary referral center. Second, AMI was diagnosed by operation, angiography and abdominal CT findings (pneumatosis intestinalis, portomesenteric gas, mesenteric arterial or venous thrombus). Third, this study could not provide clinical evidence of D-dimer, lactate or arterial gas in the differentiation between SIIH and AMI because the rates of these data were low, especially in 
SIIH patients.

\section{Conclusion}

In conclusion, our retrospective study showed that $\mathrm{SIIH}$ patients had significant maximal intestinal wall thickening, a high rate of non-involvement of the ileum and ascites as compared with AMI. According to the ROC curve analysis, the optimal cut-off value of maximal intestinal wall thickening in SIIH was greater than $10.4 \mathrm{~mm}$. The multiple logistic backward regression analysis showed that the independent predictors of acute abdomen patients with SIIH from AMI after abdominal CT were intestinal wall thickening $\geq 10.4$ $\mathrm{mm}$, non-involvement of the ileum, and ascites.

The authors state that they have no Conflict of Interest (COI).

\section{Acknowledgement}

We thank Hsin-Yi Huang from the Biostatistics Task Force, Taipei Veterans General Hospital, for statistical assistance.

\section{References}

1. Polat C, Dervisoglu A, Guven H, et al. Anticoagulant-induced intramural intestinal hematoma. Am J Emerg Med 21: 208-211, 2003.

2. Tseng CY, Chiu YH, Chuang JL, et al. How to differentiate spontaneous intramural intestinal hemorrhage from acute mesenteric ischemia. Am J Emerg Med 31: 1586-1590, 2013.

3. Abbas MA, Collins JM, Olden KW. Spontaneous intramural small-bowel hematoma: imaging findings and outcome. AJR Am J Roentgenol 179: 1389-1394, 2002.

4. Bartnicke BJ, Balfe DM. CT appearance of intestinal ischemia and intramural hemorrhage. Radiol Clin North Am 32: 845-860, 1994.

5. Balthazar EJ, Hulnick D, Megibow AJ, Opulencia JF. Computed tomography of intramural intestinal hemorrhage and bowel ischemia. J Comput Assist Tomogr 11: 67-72, 1987.

6. Chou CK. CT manifestations of bowel ischemia. AJR Am J Roentgenol 178: 87-91, 2002.

7. Tseng CY, Fan JS, Yang SC, et al. Anticoagulant-induced intramural intestinal hemorrhage. Am J Emerg Med 28: 937-940, 2010.

8. Abbas MA, Collins JM, Olden KW, Kelly KA. Spontaneous intramural small-bowel hematoma: clinical presentation and long-term outcome. Arch Surg 137: 306-310, 2002.
9. Park WM, Gloviczki P, Cherry KJ Jr, et al. Contemporary management of acute mesenteric ischemia: factors associated with survival. J Vasc Surg 35: 445-452, 2002.

10. Kougias P, Lau D, El Sayed HF, Zhou W, Huynh TT, Lin PH. Determinants of mortality and treatment outcome following surgical interventions for acute mesenteric ischemia. J Vasc Surg 46: 467474, 2007.

11. Bradbury AW, Brittenden J, McBride K, Ruckley CV. Mesenteric ischaemia: a multidisciplinary approach. Br J Surg 82: 1446-1459, 1995.

12. Oldenburg WA, Lau LL, Rodenberg TJ, Edmonds HJ, Burger CD. Acute mesenteric ischemia: a clinical review. Arch Intern Med 164: 1054-1062, 2004.

13. Birns MT, Katon RM, Keller F. Intramural hematoma of the small intestine presenting with major upper gastrointestinal hemorrhage. Case report and review of the literature. Gastroenterology $\mathbf{7 7}$ : 1094-1100, 1979.

14. Macari M, Chandarana $H$, Balthazar E, Babb J. Intestinal ischemia versus intramural hemorrhage: CT evaluation. AJR Am J Roentgenol 180: 177-184, 2003.

15. Carkman S, Ozben V, Saribeyoğlu K, et al. Spontaneous intramural hematoma of the small intestine. Ulus Travma Acil Cerrahi Derg 16: 165-169, 2010.

16. Lund EC, Han SY, Holley HC, Berland LL. Intestinal ischemia: comparison of plain radiographic and computed tomographic findings. Radiographics 8: 1083-1108, 1988.

17. Kernagis LY, Levine MS, Jacobs JE. Pneumatosis intestinalis in patients with ischemia: correlation of CT findings with viability of the bowel. AJR Am J Roentgenol 180: 733-736, 2003.

18. Knechtle SJ, Davidoff AM, Rice RP. Pneumatosis intestinalis. Surgical management and clinical outcome. Ann Surg 212: 160-165, 1990.

19. Heng Y, Schuffler MD, Haggitt RC. Pneumatosis intestinalis: a review. Am J Gastroenterol 90: 1747-1758, 1995.

20. Galandiuk S, Fazio VW. Pneumatosis cystoides intestinalis. A review of the literature. Dis Colon Rectum 29: 358-363, 1986.

21. Huang HH, Chang YC, Yen DH, et al. Clinical factors and outcomes in patients with acute mesenteric ischemia in the emergency department. J Chin Med Assoc 68: 299-306, 2005.

22. Liebman PR, Patten MT, Manny J, Benfield JR, Hechtman HB. Hepatic-portal venous gas in adults: etiology, pathophysiology and clinical significance. Ann Surg 187: 281-287, 1978.

The Internal Medicine is an Open Access article distributed under the Creative Commons Attribution-NonCommercial-NoDerivatives 4.0 International License. To view the details of this license, please visit (https://creativecommons.org/licenses/ by-nc-nd/4.0/).

(C) 2016 The Japanese Society of Internal Medicine http://www.naika.or.jp/imonline/index.html 